\title{
Dermatosis Papulosa Nigra
}

National Cancer Institute

\section{Source}

National Cancer Institute. Dermatosis Papulosa Nigra. NCI Thesaurus. Code C2984.

A benign skin condition commonly seen in dark-skinned individuals that is characterized by multiple small hyperpigmented papular lesions resembling seborrheic keratosis on the face and upper body. 